\title{
Le conflit des libertés. Thomas Paine et le débat sur la déclaration et la constitution de l'an III
}

\section{Yannick Bosc}

\section{(2) OpenEdition \\ 1 Journals}

\section{Édition électronique}

URL : https://journals.openedition.org/ahrf/2923

DOI : 10.4000/ahrf.2923

ISSN : 1952-403X

Éditeur :

Armand Colin, Société des études robespierristes

\section{Édition imprimée}

Date de publication : 1 mars 2002

Pagination : 99-105

ISSN : 0003-4436

\section{Référence électronique}

Yannick Bosc, «Le conflit des libertés. Thomas Paine et le débat sur la déclaration et la constitution de l'an III », Annales historiques de la Révolution française [En ligne], 327 | janvier-mars 2002, mis en ligne le 19 mars 2008, consulté le 23 avril 2022. URL : http://journals.openedition.org/ahrf/2923 ; DOI :

https://doi.org/10.4000/ahrf.2923 


\title{
SOUTENANCES DE THÈSES
}

\author{
LE CONFLIT DES LIBERTÉS \\ THOMAS PAINE ET LE DÉBAT SUR LA DÉCLARATION \\ ET LA CONSTITUTION DE L'AN III (1)
}

\author{
YANNICK BOSC
}

Notre étude analyse le débat sur la Déclaration et la Constitution de l'été 1795, à partir de la critique formulée par Thomas Paine dans son discours à la Convention le 19 messidor an III. Cette critique dénonce un projet de Constitution « rétrograde des véritables principes de liberté ». Nous décrivons le contenu et les justifications du projet de Constitution présenté par la Commission des onze ainsi que le débat auquel il donne lieu à l'Assemblée en les confrontant à la conception politique défendue par Thomas Paine. Les questions de la citoyenneté, de la représentation, du rapport entre état de nature et état social, entre droit matériel et droit personnel, de la relation à la Terreur, sont les axes principaux de notre recherche qui tend à mettre en évidence le conflit pohtique du libéralisme égalitaire et du libéralisme économique au cours $\mathrm{du}$ « moment thermidorien ».

Notre méthode a consisté à isoler, puis à déplier un discours choisi afin que, par ouvertures successives, il permette de saisir un ensemble. Notre démarche est analytique, donc démonstrative. La première partie est ainsi entièrement consacrée à l'intervention de Paine le 19 messidor an III à la Convention nationale. Cet Anglais qui a participé à la révolution américaine

(1) Thèse soutenue à Aix-en-Provence le 18 mars 2000, devant un jury composé de Florence Gauthier, Jacques Guilhaumou (direction), Domenico Losurdo, Jean-Clément Martin, Christine Peyrard.

Annales historiques de la Révolution française - $2002-N^{\circ} 1$ [99 à 127] 
est un homme célèbre, que l'historiographie classe parmi les modèles du libéralisme. Il est l'auteur des Droits de l'homme (1791), le plus grand «succès de librairie» de l'époque pour lequel il sera condamné par le gouvernement anglais. Élu député pour le Pas-de-Calais, il est arrêté et emprisonné au Luxembourg (27 décembre 1793). Libéré le 4 novembre 1794, il retrouve sa place de député à la Convention dans les mois qui suivent la chute des robespierristes (8 décembre 1794). Son discours du 19 messidor an III est ignoré par l'historiographie alors que c'est, à l'Assemblée, la seule critique du projet de Constitution qui est alors en débat. Notre objectif a été de montrer que ce discours et les imprimés qui lui sont connexes (Dissertation sur les premiers principes de gouvernement, Justice agraire) sont au cœur des enjeux politiques de la période. Replacé au centre du débat sur la Déclaration et la Constitution de 1795, Paine, figure insoupçonnable, victime de la Terreur et classée parmi les libéraux, bouscule ce que Jean-Pierre Faye nomme le «récit standard de l'histoire», celui qui coupe en deux la Révolution française, entre les Droits de l'homme et la Terreur.

Paine dénonce le suffrage censitaire qui, dans le projet de Constitution présenté par la Commission des onze, limite l'accès au titre de citoyen. Il estime que la propriété a remplacé les principes et l'intérêt personnel l'enthousiasme. Le projet de Constitution crée un peuple sans nom puisque une partie du peuple ne possède pas les droits de citoyen. Pour Paine, la notion de citoyenneté ne peut pas être séparée des qualités (ou droit naturels) inhérentes à l'homme puisque la citoyenneté est l'exercice de ces qualités dans la société. La conséquence est que le droit de suffrage ne peut pas dépendre de variables extérieures au droit, c'est-à-dire à l'homme : la propriété matérielle ne peut pas l'emporter sur la propriété de soi qui implique le droit à la représentation de soi. Seul le respect du principe d'égalité peut constituer un ordre social. Tout ordre social fondé sur la propriété matérielle est en fait la source du désordre. Paine dénonce l'illégalité du suffrage censitaire et assimile la Constitution de 1795 à un coup d'État parlementaire.

C'est à la suite de ce débat où il condamne l'usage de la propriété matérielle «ostensible» et le mépris de la propriété du pauvre (qui se résume souvent à la propriété de soi) que Paine rédige La justice agraire, un plan pour l'amélioration du sort des hommes. Il y expose le principe d'une « révolution dans l'état de civilisation » envisagée comme le complémentaire de la révolution du gouvernement représentatif. Selon lui, l'état dit civilisé a produit la violence et l'injustice et donc le désordre. L'état dit civilisé est dominé par la loi agraire des conquérants qui a produit la richesse et la pauvreté en dépouillant une partie de l'humanité de ce qui était avant à tous. L'état de civilisation révolutionné doit être conforme aux principes de justice représentative mais aussi de justice distributive. Paine propose donc de créer une allocation versée à toute personne, riche ou pauvre, arrivant à 
l'âge adulte ou entrant dans la vieillesse puisque tout homme a droit à une part de son héritage naturel. La situation du plus pauvre est la norme à partir de laquelle Paine conçoit un ordre social : la situation du plus pauvre dans la société ne doit pas être pire que lorsque la société n'est pas constituée. Le libéralisme de Paine fait du principe d'égalité (ou de réciprocité) le régulateur de l'ordre social.

Le discours de Paine est dirigé contre le rapport lu à la Convention par Boissy d'Anglas le 5 messidor au nom de la Commission des onze. Suivant Paine ce rapport est "rétrograde des véritables principes de liberté». Boissy d'Anglas expose un système politique (celui qui alors est dominant) dont nous faisons le contrepoint de la position de Paine. Le travail de Boissy d'Anglas est de justifier l'abandon de la Constitution de 1793 et la légitimité de la nouvelle Constitution proposée par la Commision des onze. Cette Constitution a, dit Boissy d'Anglas, pour fonction de garantir la liberté. Il construit ainsi un récit de l'expérience et de la légitimité à partir d'une histoire de la Révolution française (de 1789 à 1795). Il s'appuie sur cette narration pour expliquer les échecs des Assemblées successives qui, depuis 1789, n'ont pas su maîtriser le peuple qu'elles avaient «électrisé ». Pour Boissy d'Anglas, la Constitution de 1793 perpétue cette situation : elle est l'organisation de l'anarchie "puisque le peuple y est «constamment délibérant » et «l'opposition organisée ». Suivant Boissy d'Anglas, l'expérience de la Terreur indique que ce système de la Constitution de 1793 a été liberticide. Dès lors il faut à sa place instituer un ordre social qui puisse maintenir la liberté : celui où la propriété matérielle est une vertu. Il faut endiguer l'enthousiasme populaire et l'électrisation qui n'ont pas été maîtrisés. «Un pays gouverné par les propriétaires [dit Boissy d'Anglas] est dans l'ordre social, celui où les non-propriétaires gouvernent est dans l'état de nature ». Seuls ceux qui paient une contribution pourront être citoyens : on ne peut donner à des hommes sans propriété les droits politiques sans réserve. Les critères matériels conditionnent l'exclusion des droits politiques. L'expérience de la Terreur qui a déployé la «tyrannie de l'anarchie », au cours de laquelle le peuple s'est montré anarchiste, justifie les dégradés de l'exclusion proposés par Boissy d'Anglas (exclusion du corps social, du titre de citoyen français, de l'exercice du droit de citoyen). L'objectif de la nouvelle Constitution est d'arrêter l'impétuosité, l'émotion, l'enthousiasme, la turbulence des passions populaires mais aussi des représentants par le bicamérisme. Boissy d'Anglas reproche également aux «terroristes» et à leur ouvrage (la Constitution de 1793) la faiblesse de l'exécutif et de la centralisation. Boissy d'Anglas parle peu de la Déclaration des droits qu'il aborde succintement et tout à la fin de son discours. Elle est pourtant implicitement au centre de son propos. Pour Paine, les principes de la Déclaration sont la source à partir de laquelle il convient de penser un état social. Pour Boissy d'Anglas, la Déclaration n'est pas une loi. Ses principes 
produisent l'anarchie : l'expérience de ces «six années qui ont duré six siècles » l'a à ses yeux démontré.

Après avoir confronté les conceptions antagonistes de Paine et de Boissy d'Anglas, nous proposons un troisième angle d'attaque du débat sur les institutions de l'an III. Pour cela, nous avons établi un tableau d'une centaine de pages qui récapitule la totalité des interventions à l'Assemblée : leurs contenus, les amendements et leur devenir entre le passage en commission et la deuxième lecture. Un chiffrage permet de constater que les thèmes abordés par Paine sont prépondérants dans le débat sur la Déclaration et que les articles sur lesquels il s'appuie pour formuler sa critique sont ceux qui sont éliminés du texte en deuxième lecture.

Notre quatrième partie s'attache au débat à la Convention. Nous nous concentrons sur ce qui a été mis en avant par Paine et passé sous silence par Boissy d'Anglas : que signifie le fait de déclarer les Droits de l'homme et du citoyen en 1795 et faut-il les déclarer? Suivant les députés qui radicalisent la position de la Commission des onze, la Déclaration des droits a pu être utile au début de la Révolution quand il s'agissait d'entretenir un enthousiasme nécessaire au renversement de l'Ancien Régime. Mais une fois la République établie, une Déclaration est dangereuse. Il faut «laisser la philosophie dans les livres, là où elle n'excitera pas la guerre civile ». Daunou qui dirige les débats au nom de la Commission des onze convient qu'il eût été plus sage de la rédiger différemment en 1789. Mais l'habitude est prise. La Déclaration est devenue un point de ralliement des républicains qui est essentiel face à la menace royaliste (débarquement de Quiberon). Aussi, puisqu'elle est dangereuse et malheureusement nécessaire, il faut qu'elle soit à la fois vide et présente. Daunou justifie encore la présence d'une Déclaration en expliquant qu'il ne faut pas donner aux terroristes l'occasion de dire que l'on a «foulé aux pieds la charte des droits de l'homme». Daunou qui est un pourfendeur de la Terreur fait des terroristes les défenseurs crédibles de la Déclaration de droits. Il met ainsi en évidence la terreur de la Déclaration, incite à examiner le rapport entre Terreur et droits de l'homme et à proposer une critique du récit standard suivant lequel la période thermidorienne serait celle du retour aux droits de l'homme après la Terreur.

Par la voix de la Commission des onze, la Convention rejette la fonction normative de la Déclaration. Elle n'est pas une loi. Aussi la Déclaration que la Convention doit adopter en 1795 ne peut pas être au-dessus de la loi, ne doit pas favoriser l'enthousiasme, les principes déclarés ne doivent pas exposer le citoyen à substituer le raisonnement à la loi. Ces caractéristiques combattues sont exposées dans les principes du droit naturel tels que Paine les défend. En d'autres termes, si les droits naturels n'ont aucun caractère normatif et qu'ils restent dans les livres, alors la Déclaration ne sera pas dangereuse. Éliminés du projet qui est soumis à la discussion, les droits 
naturels reviennent avec le débat lorsque le député Defermon demande que, dans la Déclaration, figure le principe suivant lequel «tous les hommes naissent libres et égaux en droits ». Nous étudions les différentes lectures de cette proposition au sein de l'Assemblée, les arguments des conventionnels qui la défendent ou ceux qui s'y opposent. Nous examinons le trajet de cette proposition qui est adoptée en première lecture mais qui ne figure pas dans la seconde et qui est éliminée du texte final sous pression de la Commision des onze : inscrire la proposition Defermon dans la Déclaration impliquerait une autre Constitution (non censitaire); la proposition Defermon est « une arme terrible contre la Constitution » car elle est ouverte à toutes les interprétations. Or l'expérience accumulée depuis 1789 a montré le danger de la philosophie qui met en débat, qui maintient le mouvement alors qu'on recherche «le repos », qui entraîne le sujet agissant et l'enthousiasme jusqu'au « délire».

Notre cinquième partie porte sur les ajustements, les stratégies et les usages. Nous examinons tout d'abord la tension entre l'égalité et l'exclusion qui résulte des choix de la Commission des onze - suivis par la Convention ce que nous appelons l'ajustement des langages. Nous décrivons ainsi le travail d'ajustement de la Convention qui après avoir accepté la proposition Defermon en première lecture la rejette au cours de la seconde. Nous définissons ce travail d'ajustement comme le passagé d'une forme de justification à une autre, c'est-à-dire le rejet de la justification par les principes tels qu'ils sont rappelés par Paine et l'acceptation de la justification par l'expérience énoncée par Boissy d'Anglas. Le temps du travail d'ajustement est celui du débat sur la Constitution. Nous nous consacrons principalement à «l'état politique des citoyens ». Contre la critique de Paine faite au nom des principes, les conventionnels se justifient à partir des principes, c'est-à-dire du langage du droit. Ce langage du droit entre alors en tension avec le discours de l'exclusion. Seul Lanjuinais échappe à cette contradiction en développant la notion d'exclusion comme justice. À l'inverse de Paine pour lequel l'unité de mesure de l'état de civilisation est la misérable existence du pauvre, Lanjuinais fait de celui qui apporte quelque chose (au sens matériel) la mesure de l'ordre social. Lanjuinais qui expose la logique de la Constitution de 1795 indique que l'effort pour être citoyen est moins l'effort dans l'exercice de cette fonction (Paine) qu'un effort pour accéder à la fonction de citoyen. L'exercice du droit de citoyen comme base de l'ordre social (la pratique comme moyen de formation de l'homme libre) est remplacé par l'établissement de critères qui définissent ce que c'est qu'être libre pour devenir citoyen. La relation de la citoyenneté à la liberté est inversée. Notre description s'engage ensuite sur les liens problématiques entre égalité, citoyenneté et souveraineté, ce que nous nommons l'ajustement des institutions. Nous montrons que le discours de Boissy d'Anglas est décalé par rapport au texte de la Constitution qu'il est pourtant censé présenter. Il 
utilise en effet une ressource théorique inadaptée au contenu du texte constitutionnel mais qui est en revanche adaptée au travail de légitimation qui doit permettre de justifier ce texte. Le but est d'introduire artificiellement le temps de l'association : si «tous les Français » ou le peuple sont bien présents dans le discours de légitimation (Boissy d'Anglas) ils sont en revanche absents des principes d'organisation (Constitution). Dès lors, la Déclaration et la Constitution de l'an III sont des textes par lesquels le peuple («tous les Français») transfère sa souveraineté au sous-ensemble des contribuables. Ce décalage s'explique par les choix stratégiques de la Commission des onze dont les archives (nous décrivons différents états du projet présenté aux députés) valident les analyses que nous avons menées à partir du débat à la Convention ainsi que la fabrication du discours de légitimation prononcé par Boissy d'Anglas. Les papiers de Daunou - qui a la haute main sur les travaux de la Commission - indiquent également que la Commission désigne les principes du droit naturel et la question de la propriété comme les points de débat à éviter. Ils confirment la volonté de rupture avec les principes énoncés en 1789 ou 1793.

Nous nous trouvons dès lors face à un enchevêtrement où se mêlent le récit construit par les thermidoriens et celui des historiens - qui souvent le reprennent sans distance - récits dans lesquels la violence, la justice, le droit, la Terreur occupent une place déterminante. Nous décrivons l'usage que le discours de légitimation fait de la Terreur et de l'insurrection populaire (prairial) comme repoussoirs universels afin d'établir une continuité entre le 9 Thermidor, le retour de la liberté et la Constitution de 1795. Or, la figure de Thomas Paine dénonce la fluidité apparente de cet enchaînement. Paine est une victime de la Terreur et il le rappelle au début de son intervention du 19 messidor. Son travail politique consiste à souligner que la Commission des onze utilise les misères et les crimes (le sang de la Terreur) pour mettre à mal la liberté en touchant à l'égalité. Il dit que même enfermé au Luxembourg il gardait espoir puisque le principe d'égalité existait encore. À ses yeux, l'événement n'est pas le 9 Thermidor sur lequel le discours officiel focalise l'attention - puisqu'il est la nouvelle source de la légitimité de la Convention qui a alors fait sa révolution. Pour Paine, l'événement réside dans la rupture fondamentale qui consiste à tuer la liberté en limitant la citoyenneté. À la suite de son discours du 19 messidor, un journal publié par un de ses proches (Louvet) n'hésite pas à identifier Paine à Robespierre : « leur système est le même ». Pour les contemporains, en l'an III, il n'y a pas de différence entre les idées des deux hommes que l'historiographie oppose. Ils sont tous les deux des libéraux-égalitaires.

Nous abordons enfin les enjeux pour notre siècle en montrant que de nos jours des économistes et des philosophes exhument la figure de Thomas Paine. Ils constatent en particulier «l'étrange modernité » et l'efficacité du principe d'allocation universelle que Paine élabore à partir de son travail 
critique de la Constitution de 1795. Plus encore ils retrouvent, face à l'exclusion qui nous est contemporaine, les termes du débat de l'an III sans le connaître ni même se référer à la Révolution française - et c'est bien sûr cette conjonction qui est intéressante. Le diagnostic posé par Paine sur une conception de l'ordre social et de la République qu'il combattait il y a deux cents ans serait ainsi plus que jamais d'actualité. 